\title{
Research on Cloud Data Center System Design in Intelligent Community
}

\author{
Liu Tao ${ }^{1, \text { a }}$ \\ ${ }^{1}$ Chongqing College of Electronic Engineering, Chongqing, China,401331 \\ a15823118520@139.com
}

\section{Keywords: Research, Cloud Data Center System, Design, Intelligent Community Computer}

\begin{abstract}
With the development of computer technology, communication technology and Web technology, intelligent building has been widely used and accepted for many years. In this paper, the key technology of cloud data center system design is analyzed. At the same time, the realization principle of the system and each function module are introduced in detail and the system implementation work is also discussed.
\end{abstract}

\section{Introduction}

With the advent of the 21st century quietly, great changes are taking place quietly in our society at the same time. Living conditions are changing more and more. Along with the development of the national economy and the continuous improvement of people's living standard, people not only meet the needs of simple residential areas, entertainment needs, service, security and information consultation and so have an urgent requirement. The application of intelligent network in residential building has brought a new face and the concept of intelligent community also came into being. In the process of globalization, electronic information, network communication technology, information processing technology and its economic development, the information technology in the process of moving forward, the Chinese society of information technology development and application of the economy, science and technology, culture, etc. The impact of intelligent building plays a very important social role.

The so-called "intelligent building" is based on the advanced integrated computer technology and communication technology, to manage the construction of a building automation management to achieve the building electrical, lighting, air conditioning, anti-theft control, disaster prevention and transportation equipment, The office automation operation realization intelligent control management, constructs a modern management residential district.

Intelligent community is the social and economic information of the international, highly integrated multi-disciplinary high-tech inevitable product. A large number of high-tech-intensive technology, advanced equipment, the most advanced multi-functional running applications, set video telephony, video intercom, e-mail, wireless network, satellite communications, international communication computer network, environmental control, intelligent security, The information superhighway can effectively be an integrated system to meet different social needs, fully modernized residential area with strong vitality and broad vision and intelligent community development.

\section{The Related Technology}

The HTML Language. HTML (Hypertext Markup Language) is a language-based file and that is an HTML Web site. Powerful HTML format support embedded data file language allows language structure, it is a common development language global information website, which is characterized by: 1. Language simple hypertext markup language HTML application flexibility and interest, the language is simple, the structure. 2. Language has a very good scalability. The sub-class elements of the path design HTML language is to improve its operation, a wide range of applications effectively ensure the scalability of the language. 3. Platform independence PC platform in a wide range, you can use the HTML language can also be applied to the MAC address and other machines and it has become a popular web page design and development of another major reason. 
ASP.NET Technology. ASP.net is fundamentally different from traditional HTML. The system is developed by using the object-oriented ASP.net programming model, which can achieve the system maintainability and security better. In the object-oriented language, the main business logic for the specific implementation of the system, the implementation of the program is usually used ASP.net development technology, through the function of the dynamic injection of code to isolate the business logic and view the display, reducing the level. The system view layer mainly realizes the interaction with the user. Usually, the server-side scripting ASP.NET website of the active environment system of the object-oriented language system can run and generate interactive, dynamic, high-performance Web service s application.

ASP.net development in the field, the traditional design based on the database application is a relationship-oriented - the real object-oriented direction in dealing with the relevant database process has not been achieved, this type of design is only around the object relationship between the mapping with a simple representation of accessing persistence data. In the application ASP.net, generally use DBC and other common technologies to create such a persistent layer. To the persistence of the most commonly used data layer ASP.net method, for example, used in enterprise-class development, often use DAO to access the package data, then the package data in BO (Business Object) unified call. But the DAO object closely coupled with the SQL language, it does not support inheritance and polymorphism, the system maintainability is greatly reduced; the other hand, EJB, it must take up additional EJB containers (usually using the application server) to expand, More do not support inheritance and polymorphism. Therefore, these traditional persistence solutions exist many problems let us have this idea: We try to use Hibernate packaging database, so that it can be directly object-oriented, this means not only solve these problems, but also to make cumbersome and repeat the work easy Up, although the technical content of database programming is not high, but the superiority of this approach is exposed. $1.3 \mathrm{~B} / \mathrm{S}$ Mode Combining the advantages and disadvantages of the above B / S and C / S, if I only B / S or C / S mode in the intelligent building network system, we can satisfy the current demand to the utmost extent. This combination of the two methods, not only to meet the requirements of good high-security systems, massive data processing capabilities, and strong interaction of the same data, high-security systems only use the model. B / S and C / S mixed mode model structure.

SQL Server Database Technology. In the database which we mainly use SqlServer2005 database, SqlServer2005 we mainly introduce SqlServer database, SqlServer database is Microsoft launched the database, it has the following characteristics: the client / server software architecture structure, through the request and service mode and database communication ; Graphical user interface for data maintenance and management, the database system management and data manipulation more simple and convenient; provides a rich programming interface, the user can be graphical or command-line based on the way to manage, Has a very good scalability; has a powerful Web-based analysis, support for distributed partition views and other excellent features, so this project is mainly based on SqlServer 2005 database, which provides us with a very good data support system service.

\section{The Detailed Design of the System}

As the management mode of the property management center is also more and more intelligent development in the area of building security has been a large number of inputs into a variety of security, equipment, fire and other management systems, but because the various subsystems are independent of each other to use more trouble, Need to repeatedly log on multiple systems to complete all the operations, so a great waste of time and increased efficiency, it is necessary to the district of all the subsystems of the organic unity of management, thereby enhancing the Staff efficiency, the entire property management center to reduce economic costs, intelligent community centralized management system, the main functions of the family information service system, residential automation systems, residential property management system, system management and other subsystems. Intelligent plot centralized management system design principles and design goals is particularly important, it determines the life cycle of a system, but also to facilitate the 
project's late upgrade work, so the system must follow the following design principles:

The modular design: due to the needs of pre-survey document data redundancy, and more confusing, only a large number of data analysis documents in accordance with the requirements of the design principles into a detailed summary of the various independent modules, according to similar types of functional modules integrated storage together constitute a small sub-function module. At the same time for the module to develop input and output values, the operation of each module, the module content of the property values, the convenience of the module, the use of security and other aspects of planning.

The good database design style: database management system in strict accordance with the definition of the database design paradigm for data planning and design, and follow the basic requirements of structured query language table creation, view management, index management, storage process management And a series of design principles.

The completed information query statistics: the basic function of property management is for the development of grass-roots personnel custom development, but the leadership of management will be concerned about the basic operations of the business, it must create query statistics function modules can be summarized as a leader at the end of the year- report statistics and decision support.

The post-maintenance: the development of the Intelligent Community centralized monitoring system in the system architecture design must be considered in the latter part of the system maintenance work must be in the system design must be structured in accordance with the design method for the design, in order to add new functions, Operation, thereby enhancing the system utilization and system expansion functions and other effects.

\section{The Implementation of the System}

The Home Information Service System. The home information service mainly provides the self-service management platform for the home users in the community, and provides various information consulting services for the local community users, and mainly uses the remote teaching management module, the online entertainment management module, the online shopping management module, the online consultation management module , Remote medical management and management module, as long as equipped with the necessary equipment, such as the system will be equipped with network television, routers and other important equipment, you can back-end service center entry program broadcast to the community for each home user to listen.

The Realization of the Intelligent Home. Home is an important basic equipment for various home users, each home to the home users play a supreme family service work, only the home of the intelligent home for effective management to make a variety of smart home play to the highest role in order to better For the user to improve the high service work, the community's smart home is to use the Internet network to connect to the community of public equipment such as central air conditioning, residential lighting and other facilities and home automation equipment such as lighting systems, curtains system for remote control Wait.

The Access Control System. The access control system consists of property management server, ordinary computer (WEB client), magnetic card reader, IC card, intelligent door and so on. When the input password is incorrect, the alarm system of the access control system automatically alarm, Property management center also get the alarm information in order to facilitate the immediate control and follow-up investigation and tracking. When the illegal personnel alarm, the system automatically start the district's monitoring equipment, real-time capture of the illegal personnel for the late police investigation to provide technical decision support role.

The Closed-Circuit Monitoring System. Closed-circuit monitoring system is mainly for the construction of a comprehensive multi-directional security monitoring, will be important in the channel mouth, parking lot, the entrance, the installation of important facilities, video camera, the monitoring center can clearly see the scene of each important place for Timely, but also can be used as other alarm system alarm notification after the video linkage operation, that is, where the video camera immediately transferred to the site for video recording.

The Public Device Management. Public equipment management is mainly on the effective 
management of intelligent devices in the district, such as the lighting system can be on the campus lighting system registration, maintenance, replacement, scrap and other related records; the district's water tank, temperature and humidity, with UPS, air conditioning, fire and other real-time monitoring, such as the current water tank, the remaining water on-line monitoring in order to facilitate the development of the entire district owners have water shortages, thus affecting the quality of life of residential property owners; In order to reduce the occurrence of security incidents or for a variety of events recorded in the latter part of an effective search and information acquisition function, the main interface in the public equipment operation is mainly divided into two columns to display the contents of the left shows a brief device information, a property center or a If you click on the device tree on the left side and the device tree on the left side will be displayed on the right, you can find the details of each device under the device type automatically. The device tree is displayed in the tree structure.

Realization of Property Charge Management. Service fee management is the property management center of the user's various information and billing information corresponding to the maintenance of data and water and electricity within the district to settle charges, but also through the charge management to complete the user's cost to pay, and the user's Payment to save, and print the corresponding water and electricity charges to pay the receipt. In the realization of the operation of the property charge management interface, should be considered in many ways, in an interface to display the main property charges information, but also a quick display of various household user information and user-specific parameters, or monthly charges, and now Details of the charges.

\section{Conclusion}

Intelligent residential area cloud data center system as a high-tech product that makes live more convenient, improve the quality of life and promote energy-saving, it has gradually entered the homes of ordinary people. The construction of cloud data center system is based on the intelligent technology, computer technology, communication technology, information processing technology, IC card technology and intelligent control technology. It provides a convenient, comfortable, efficient and energy-saving residential environment for residential users in the intelligent building community.

\section{References}

[1] Huifang Zhou: Automation of Electric Power Systems, Vol. 6 (2014) No 53, p.25-26

[2] Hongli Zhang: Fiber and Cable and Their Applications, Vol. 12 (2015) No 27, p.74-76

[3] Qin Guo: Journal of Nankai University, Vol. 1 (2012) No 33, p.11-14

[4] Jieming Liu: Wuhan Ship Vocational and Technical College, Vol. 3 (2011) No33, p.121-124

[5] Wu Gongyi, Cao Yong: Chongqing Polytechnic College, Vol. 5 (2012) No17, p.53-54 